\title{
VILOSITE DE ETIOLOGIA DESCONHECIDA EM PLACENTAS DE GESTAÇÕES COM HIPERTENSÃO ARTERIAL E DE GESTAÇÕES COM RECÉM-NASCIDOS PEQUENOS PARA A IDADE GESTACIONAL
}

Albina Milani Altemani*, Adriana Rocha Gonzatti

Trabalho realizado no Departamento de Anatomia Patológica da Faculdade de Ciências Médicas da UNICAMP, Campinas, SP

RESUMO - OBjEtivos. Analisar a freqüência da vilosite de etiologia desconhecida (VED) e suas características histológicas em placentas de gestaçôes com hipertensão arterial materna e de gestações com recém-nascidos (RN) pequenos para a idade gestacional.

Métodos. Foram estudadas $2 / 3$ placentas de mães e RN sem evidências clínicas ou sorológicas de infecção. Estas placentas foram subdivididas conforme a condição materna em: normotensas - |5| casos, doença hipertensiva específica da gravidez (DHEG) - 37 e hipertensão crônica - 25 e, de acordo com o peso do RN, em: pequenos (PIG)- 38 casos e adequados para a idade gestacional (AIG) - 175. Destas placentas, 8I pertenciam a uma amostra aleatória, que foi utilizada para determinar a freqüência de VED na população estudada. Foram retirados oito fragmentos do parênquima placentário e as secções histológicas foram coradas por HE. Para análise estatística foram utilizados os testes de qui-quadrado e exato de Fisher, sendo $p<0.05$ considerado significante.

Resultados. A freqüência de VED nas placentas foi de $30,8 \%$ na amostra aleatória, $39 \%$ nas normotensas, $29,7 \%$ nas gestações com
DHEG, 32\% nas hipertensas crônicas, 34,2\% nos RN PIG e 37,1\% nos RN AIG. Nas placentas de gestações com hipertensão arterial predominou a vilosite com componente parenquimatoso (DHEG $27 \%$, hipertensão crônica - 28\%). Este tipo de vilosite também foi a mais comum nas placentas dos RN PIG (31,5\%). Em contraste, a vilosite basal sem o componente parenquimatoso predominou nas placentas de normotensas (16,5\%) e de RN AIG (14,8\%). A maioria das vilosites era de intensidade leve.

CoNcLusóes. Na população estudada, a frequiência de VED é alta, em torno de $30 \%$. A VED ocorre com freqüências semelhantes em placentas de normotensas, de gestações com DHEG ou hipertensão crônica e em RN PIG e AIG, porém a vilosite basal sem o componente parenquimatoso é mais comum em normotensas e RN AIG. É possível que este tipo de vilosite seja resultante de uma estimulação antigênica diferente daquela da vilosite com componente parenquimatoso.

UnITERMOS: Placenta. Vilosite. Hipertensão arterial. Recém-nascido pequeno para a idade gestacional.

\section{INTRODUÇÃO}

A vilosite é caracterizada pela presença de infiltrado inflamatório no estroma da vilosidade placentária ${ }^{1,2}$ e é classificada quanto à etiologia em conhecida e desconhecida'. A primeira é, na maioria dos casos, a expressão placentária da infecção fetal por via hematogênica causada por vírus, bactéria ou parasita ${ }^{\prime, 3}$ Para a vilosite de etiologia desconhecida (VED) os principais fatores causais propostos são infecção materna não diagnosticada e reação imune da mãe contra os tecidos placentários ${ }^{1,4,5}$. A VED é a mais comum das vilosites, porém sua incidência varia em diferentes países e são aventados três fatores para explicar es-

*Correspondência:

Deptode Anatomia Patológica FCM/Unicamp

CaixaPostal6111

13081-970-Campinas-SP sas diferenças: número de fragmentos examinados por caso, critérios histológicos para o diagnóstico de vilosite e a população da qual a placenta é obtida ${ }^{6,7,8}$. Em relação ao fator população, as mais altas freqüências de VED tem sido descritas na América do Sul4,9. Clinicamente, a VED pode estar associada ao recém-nascido pequeno para a idade gestacional (PIG), porém a freqüência relatada desta associação é muito variável $(7,5 \%$ a $86 \%)^{3,4,10,11}$ e há controvérsias quanto à sua associação com a doença hipertensiva específica da gravidez (DHEG) $)^{11,12,13,14}$.

A natureza da associação entre VED e peso fetal diminuído não é clara, sendo que as principais hipóteses são: a) diminuição da quantia de parênquima placentário útil pela vilosite, com conseqüente diminuição da transferência de nutrientes para o feto, b) o agente infeccioso (ainda desconhecido, porém provavelmente viral) causaria infecção na placenta e no feto e, devido a esta última, ocorreria inibição da síntese de DNA fetal ' . Em relação à doença hipertensiva materna, Ruschoff et al. ${ }^{13}$ sugeriram que a isquemia útero-placentária poderia participar da patogênse da VED, pois encontraram uma maior freqüência de VED em placentas de gestações com pré-eclâmpsia. Salafia et al. ${ }^{12}$ relataram uma associação semelhante, porém outros autores ${ }^{11,14}$ não compartilharam tal observação. Fox', entretanto, ressalta que a hipercelularidade vilosa secundária à isquemia útero-placentária é encontrada freqüentemente próxima dos enfartes e pode ser histologicamente confundida com a VED.

Os objetivos do nosso trabalho são analisar a freqüência da VED e suas características histológicas em placentas de gestações com hipertensão arterial materna e de gestações com recém-nascido (RN) pequeno para a idadegestacional (PIG). 


\section{Métodos}

Primeiramente foram coletadas 132 placentas, no período de um ano, as quais foram selecionadas conforme a condição da pressão arterial materna: normotensa-79 casos, doença hipertensiva específica da gravidez (DHEG)29 casos e hipertensão crônica - 24 casos. Foram critérios de inclusão neste grupo: recémnascido vivo, mãe e RN sem evidências clínicas ou sorológicas de infecção, pré-natal completo com início antes de 20 semanas de gestação, informação adequada sobrea pressão sangüínea materna e ausência de malformações fetais. Todos os RN foram subdivididos conforme 0 peso ao nascimento, sendo considerados pequenos para a idade gestacional quando o peso em relação à idade gestacional estava abaixo do percentil I0 na curva de Lubchenco et al. ${ }^{15}$. A idade gestacional foi determinada pelo método de Capurro ${ }^{16}$. Posteriormente, foram coletadas 81 placentas consecutivamente, as quais eram provenientes dos dois primeiros partos realizados entre 08:00 e 17:00 horas de segunda à sexta-feira. Esta amostra foi denominada de aleatória, sendo critérios de exclusão: feto morto, mãe e/ ou RN com evidências clínicas ou sorológicas de infecção e malformação fetal. Destas placentas, 72 eram de gestações com pressão arterial normal e nove com hipertensão arterial (oito com doença hipertensiva específica da gravidez e um com hipertensão crônica), sendo que 77 dos RN apresentavam peso ao nascimento adequado para a idade gestacional (AIG) e quatro pequeno para a idade gestacional.

A distribuição final das 213 placentas estudadas ( 132 iniciais mais 8 I da amostra aleatória) é a seguinte: a) conforme a condição materna: normotensas - I5I casos, DHEG - 37 e hipertensão crônica - 25, b) conforme o peso do RN: PIG- 38 casos e AIG - I75. DHEG foi diagnosticada quando a pressão sangǘnea materna era igual ou maior a $140 / 90$, no mínimo em duas tomadas a partir de 20 semanas de gestação. Em 19 das 37 pacientes com DHEG o nível deácido úrico sérico foi medido e estava elevado em todas. Proteinúria acima de $0,3 \mathrm{~g} / 24$ horas estava presente em $27 \mathrm{pa}-$ cientes. O diagnóstico de hipertensão crônica foi baseado na presença de pressão sangüínea maior ou igual a $140 / 90 \mathrm{mmHg}$ antes da gravidez ou presente antes de 20 semanas de gestação e persistindo depois do puerpério.

\begin{tabular}{cccccc}
\hline \multicolumn{5}{c}{ Tabela I - Distribuição dos padróes anatomopatológicos da vilosite de etiologia } \\
desconhecida na placenta, de acordo com a síndrome hipertensiva materna \\
\hline NT & VILOSITE AUSENTE & VP $(\%)$ & VB $(\%)$ & VPB $(\%)$ & TOTAL \\
DHEG & $92(60,9)$ & $20(13,2)$ & $25(16,5)$ & $14(9,2)$ & $|5|$ \\
HC & $26(70,2)$ & $6(16,2)$ & $I(2,7)$ & $4(10,8)$ & 37 \\
\hline
\end{tabular}

Teste exato de Fisher $P=0,211$

NT:normotensa

DHEG: doençahipertensiva específica da gravidez

HC: hipertensão crônica

VP:vilositeparenquimatosa

VB:vilositebasal

VPB:vilositemista(parenquimatosaebasal)

As placentas, imediatamente após a dequitação, foram fixadas em balde contendo formalina a 10\% por 24 horas. Após exame macroscópico, elas foram clivadas e de todas foram retirados: a) Oito fragmentos do parênquima placentário sem alterações macroscópicas (tais como, enfarte, trombos, cistos, etc) compreendendo toda espessura deste (da superfície materna à fetal) e com tamanho de cerca de $0,5 \mathrm{~cm}$ de largura por 0,3 $\mathrm{cm}$ de espessura, b) Um de membrana e um de cordão umbilical. O número de fragmentos examinados por placenta foi baseado num estudo nosso prévio sobre VED (Altemani ${ }^{19}$ ). Todas as secções histológicas foram coradas por hematoxilina e eosina e examinadas pelo mesmo observador (AMA).

Vilosite foi diagnosticada quando havia nítido infiltrado inflamatório no estroma das vilosidades coriais (Fig. I). Em relação à distribuição das lesões inflamatórias no parênquima placentário, as vilosites foram classificadas, segundo Altshuler e Russell ${ }^{3,10}$, em: vilosite basal, vilosite parenquimatosa e padrão misto (basal e parenquimatosa na mesma placenta). A intensidade do processo inflamatório foi subjetivamente graduada em leve, moderada e intensa, conforme a quantidade de vilosidades acometidas. Para analisar estatisticamente as correlações de freqüência foi utilizado o teste de qui-quadrado. Sempre que necessário, devido ao pequeno número da amostra analisada ou a freqüência esperada menor do que cinco, 0 teste do qui-quadrado foi substituído pelo teste exato de Fisher, sendo que $p<0.05$ foi considerado significante. A freqüência de VED detectada na amostra aleatória foi con- siderada representativa da freqüência da lesão na população examinada neste serviço. O consentimento formalizado de participação no estudo pelas pacientes não foi necessário, pois os dados foram retirados dos prontuários clínicos e não se utilizou identificação pessoal.

\section{Resultados}

A freqüência de VED nas placentas foi de: $30,8 \%$ (25/8I) na amostra aleatória, $39 \%$ (59/ I5I) nas normotensas, 29,7\% (II/37) nas gestações com DHEG, 32,0\% (8/25) nas hipertensas crônicas, 34,2\% (I3/38) nos RN PIG e 37,I\% (65//75) nos RN AIG. Estas diferenças de freqüência entre os grupos não foram estatisticamente significantes.

$\mathrm{Na}$ amostra aleatória, a maioria das VED era de intensidade leve (67,7\% dos casos), sendo que $40 \%$ apresentavam padrão parenquimatoso, $36 \%$ basal e $24 \%$ mista.

Nas placentas de mães normotensas, a maioria das vilosites era de intensidade leve ( $71,1 \%$ ), sendo $25,4 \%$ moderada e $3,3 \%$ intensa. Neste grupo predominou a vilosite basal isolada (25/I5I - 16,5\%) (Tabela I).

Nas placentas de gestações com DHEG, a vilosite parenquimatosa foi a mais freqüente (6/37 - 16,2\%) enquanto que a vilosite basal isolada foi a menos comum ( $1 / 37-2,7 \%)$ (tabela I). Somando-se as vilosites com componente parenquimatoso (parenquimatoso isolado mais mista) a diferença foi estatisticamente significante $(p=0,0444)$ em relação a vilosite basal isolada ( $10 / 37-27 \%$ versus $I / 37$ - 2,7\%). Quanto à intensidade da vilosite, observamos que este grupo foi mais acometido por vilosites moderada e intensa $(54,4 \%)$ 


\begin{tabular}{lccccc}
\hline \multicolumn{5}{c}{$\begin{array}{c}\text { Tabela 2 - Distribuição dos padrões anatomopatológicos da vilosite de etiologia } \\
\text { desconhecida na placenta de gestações que apresentaram recém-nascidos } \\
\text { pequenos para a idade gestacional e adequados para a idade gestacional }\end{array}$} \\
\hline VILOSITE AUSENTE & VP $(\%)$ & VB No(\%) & VPB $(\%)$ & TOTAL \\
RNPIG & $25(65,7)$ & $9(23,6)$ & $I(2,6)$ & $3(7,8)$ & 38 \\
RNAIG & $110(62,8)$ & $20(11,4)$ & $26(14,8)$ & $19(10,8)$ & 175 \\
\hline
\end{tabular}

Teste exato de Fisher $\mathrm{P}=0,053$

PIG: pequeno paraaidade gestacional

AIG: adequado paraaidade gestacional

VP:vilositeparenquimatosa

VB:vilositebasal

VPB: vilositemista(parenquimatosaebasal)

Figura I - Vilosite de etiologia desconhecida: observa-se nítido infiltrado
inflamatório no estroma da vilosidade (HE X 300)

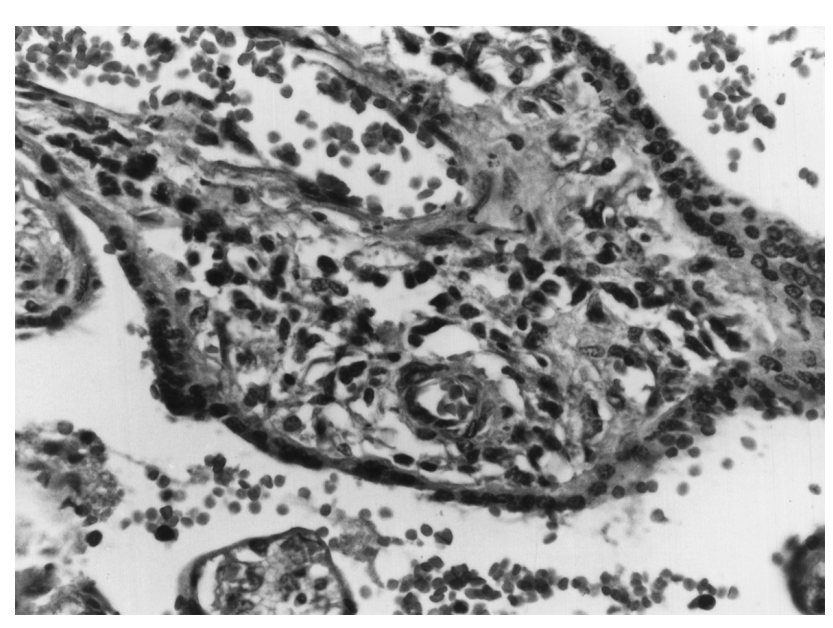

do que os outros (normotensa - 28,8\%, hipertensão crônica-37,6\%).

Nas placentas de gestações com hipertensão arterial crônica, a vilosite com padrão misto (parenquimatoso mais basal) predominou (4/25 - $16 \%$ ) e a vilosite basal isolada foi a menos freqüente (I/25 - 4\%) (Tabela I). Somandose as vilosites com componente parenquimatoso (parenquimatoso isolado mais mista) a diferença não foi estatisticamente significante $(p=0,137)$ em relação a vilosite basal isolada (7/25 - 28\% versus I/25 - 4\%). Em relação a intensidade, $62,5 \%$ eram de intensidade leve, $25 \%$ intensa e $12,5 \%$ moderada.

A freqüência dos RNPIG nos três grupos maternos estudados foi $14,5 \%$ entre as normotensas, $32,4 \%$ nas gestações com DHEG e $16 \%$ nas gestações com hipertensão crônica. Nas placentas dos RN PIG, a vilosite parenquimatosa foi a mais freqüente $(9 / 38$ $23,6 \%$ ) e a basal isolada a mais rara (1/38 - 2,6\%) (Tabela 2). Somando-se as vilosites com componente parenquimatoso (parenquimatoso isolado mais mista) a diferença foi estatisticamente significante ( $p=$ 0,02777 ) em relação a vilosite basal isolada (I2/38-31,5\% versus I/38-2,6\%). Avilosite de intensidade leve foi a mais freqüente $(69,2 \%)$, seguida daquela de intensidade moderada (30,7\%). Não encontramos nenhum caso de vilosite intensa. Nas placentas dos RN AIG, contrastando com aquelas dos RN PIG, predominou a vilosite basal isolada (26/65 $14,8 \%$ ) (Tabela 2). Em relação a intensidade houve predomínio da lesão leve $(66,1 \%)$, seguida da moderada $(24,6 \%)$ e intensa $(9,2 \%)$.

\section{Discussão}

A freqüência relatada de VED (tanto em gestações normais como anormais) é variável em diferentes países ( $2 \%$ a $26 \%)^{3,4,7,17,18}$ e as hipóteses propostas para explicar essa variabilidade são a) fatores socioeconômicos, ambientais e étnicos da população estudada, b) número de fragmentos examinados, c) critérios histológicos para o diagnóstico de vilosite $^{1,6,7,8}$. Em relação aos critérios histológicos para o diagnóstico de vilosite tivemos o cuidado de diagnosticá-la apenas quando havia um nítido infiltrado inflamatório no estroma da vilosidade. Para evitar um excesso de diagnóstico de vilosite por hipercelularidade não inflamatória do estroma, em todos os nossos casos, o infiltrado inflamatório continha células com características de linfócitos, além de macrófagos e células estromais. Outro cuidado tomado em nosso estudo foi em relação ao número de fragmentos examinados por placenta, visto que geralmente somente $2 \%$ a $4 \%$ do parênquima viloso apresenta infiltrado inflamatório na VED' e, portanto, quanto mais representado for, maior a chance de encontrar a vilosite. $O$ número de fragmentos examinados por nós (oito) é semelhante ao de Labarrere et al. ${ }^{4}$ (seis), na Argentina.

Para determinarmos a freqüência de VED na população que freqüenta o nosso hospital, e que pertence a um grupo socioeconômico de baixa renda, analisamos 81 placentas, que correspondiam aos dois primeiros partos do dia. $A$ freqüência deVED nestaamostrafoide $30,86 \%$ e é alta quando comparada com aquelas relatadas por KnoxeFox ${ }^{7}(13,6 \%)$ e Russell ${ }^{10}(7,6 \%)$ na Inglaterra e Austrália, respectivamente, porémé próxima à de Labarrerre et al. ${ }^{4}(26 \%)$ na Argentina, quando esses autores utilizaram um grupo semelhante ao nosso. Provavelmente, isto se deve, em parte, ao grande número de fragmentos examinados por placenta, por nós (oito) e Labarrere et al. ${ }^{4}$ (seis), embora não se possa afastar totalmente a influência de uma maior incidência de infecções, que é esperado ocorrer em população de baixa renda ${ }^{6}$, que constituia maioria da população examinada neste serviço. Entretanto, em relação a estaúltima possibilidade, num estudo anterior ${ }^{19}$, não detectamos níveis elevados de lgM (um monitor não específico de infecção intra-uterina) no sangue do cordão umbilical, apesar da alta freqüência de VED na nossa amostra. 
Em relação à associação da VED com a hipertensão materna, seja específica da gravidez ou crônica, não encontramos uma diferença significante na freqüência de vilosite entre estes dois grupos e os das normotensas. A análise da literatura mostra que há discordâncias sobre essa associação. Salafia et al. ${ }^{12} \mathrm{e}$ Ruschoff etal. ${ }^{13}$ relataram uma maior freqüência de VED em placentas de mães com préeclâmpsia, sendo que Ruschoff etal. ${ }^{13}$ sugerem que a isquemia útero-placentária pode participar da patogênse da VED. Entretanto, a ausência de associação significante entre VED e DHEG neste trabalho, e compartilhada por outros autores ${ }^{11,14,20}$, não apóia essa hipótese. Lesões histologicamente semelhantes à VEDe secundárias à isquemia útero-placentária foram relatadas por $\mathrm{Naeye}^{21}$ e Altshuler ${ }^{22}$, porém concordamos com Fox' que aVED pode ser diferenciada destas alterações se a diagnosticarmos apenas quando houver um infiltrado inflamatório indubitável e não somente hipercelularidade do estroma.

A freqüência de VED em placentas de recém-nascidos pequenos para a idade gestacional relatada na literatura varia de $7,5 \%$ a $86 \%^{3,4,11,23,24}$, sendo que a maioria está em torno de $30 \%$ '. Na nossa amostra, a freqüência de VED entre os RN PIG foi de $34,2 \%$, porém não diferiu significantemente da freqüência encontrada entre os RNAIG (37,I\%). Uma possibilidade para explicar essas freqüências semelhantes de VED nestes dois gruposé que, na nossa amostra, o grupo dos RN PIG era constituído predominantemente por RN com peso não muito baixo, ou seja, próximo do limite normal.

Apesar de não encontrarmos uma correlação positiva entre a freqüência de VED e hipertensão materna, a distribuição dos tipos de vilosite e sua intensidade neste grupo difere daquelas das normotensas. No nosso material, a vilosite basal isolada tem uma freqüência elevada, quando consideramos os grupos das normotensas e dos RN AIG ( $16,5 \%$ e I4,8\%, respectivamente), porém ela é baixa nas gestações com DHEG ou hipertensão crônica $(2,7 \%$ e $4 \%$, respectivamente) e nos RN PIG (2,6\%). Acreditamos que a vilosite basal isolada possa ser expressão de uma estimulação antigênica diferente daquela da vilosite parenquimatosa, que é mais freqüente em mães hipertensas ou com RN PIG e poderia corresponder a um ataque imune materno contra os tecidos fetais, como proposto por Labarrere et al. ${ }^{4,5}$. Pelos nossos achados (freqüente em $\mathrm{RN}$ com peso adequado para a idade gestacional e mães normotensas), a vilosite basal isolada aparentemente não interfere na nutrição fetal e é um evento comum em gravidez normal. Entretanto, nos casos em que a vilosite basal ocorre junto com a parenquimatosa, as duas poderiam ser resultado de um mesmo estímulo, infeccioso ou não, que se distribui aleatoriamente na placenta.

Em relação à intensidade da VED nos grupos das mães com hipertensão, específica ou não da gravidez, observamos uma maior freqüência de vilosite moderada e intensa, do que entre as normotensas (DHEG-54,5\%, hipertensão crônica-37,5\%, normotensas-28,8\%). Este achado sugere que a isquemia placentária poderia aumentar a permeabilidade do trofoblasto às células maternas, que são primariamente atraídas por um estímulo antigênico (infeccioso ou não) para o estroma viloso. A grande maioria das células inflamatórias na VED é de origem materna ${ }^{25,26}$.

\section{Conclusóes}

Na população estudada, a freqüência de VED é alta, em torno de $30 \%$. A VED ocorre com freqüências semelhantes em placentas de normotensas, de gestações com DHEG ou com hipertensão crônica e em placentas de RN PIG e AIG, porém a vilosite basal sem o componente parenquimatoso é mais comum em normotensas e RN AIG. É possível que este tipo de vilosite seja resultante de uma estimulação antigênica diferente daquela da vilosite com componente parenquimatoso.

\section{SUMMARY}

VILLITIS OF UNKNOWN ETIOLOGY IN PLACENTAS OF PREGNANCIES WITH HYPERTENSIVE DISORDERS AND OF SMALL-FORGESTATIONAL-AGE INFANTS

BACKGROUND. The objectives of this study are to analyze the frequency and the histopathological features of the villitis of unknown etiology (VUE) in placentas of pregnancies with hypertensive disorders and of small-for-gestationalage infants (SGA).

METHODS. Two hundred and thirteen placentas from pregnancies without clinical or laboratorial evidence of infection were studied. These cases were subdivided according to: a) maternal condition in: non-complicated pregnancy (NCP)- 15I cases, pregnancy-induced hypertension (PIH)- 37 and sustained chronic hypertension (SCH)- 25 and b) newborn weight in: small for gestational age (SGA)- 38 cases and adequate for gestational age (AGA)- I75. Of these placentas, 81 belong to the random sample, which was used to determine the frequency of VUE in the population studied. Eight blocks were taken from placental parenchyma and the histological sections were stained with hematoxylin and eosin. Frequency tables of categorical data were analyzed using the chisquare test and Fisher test; statistical significance was considered for $p<0.05$.

RESULTS. The frequency of VUE in the placentas was $30.8 \%$ in the random sample, $39 \%$ in NCP, $29.7 \%$ in PIH, 32\% in SCH, $34.2 \%$ in SGA infants and $37.1 \%$ in AGA infants. Placentas of pregnancies with hypertensive disorders were more affected by villitis with parenchymatous component (PIH-27.0\%, SCH-28.0\%). This lesion was also the predominant villitis in the placentas of the SGA infants (3I.5\%). In contrast, in placentas of NCP and AGA infants, the principal kind of villitis was basal, not associated to a parenchymatous component (16.5\% and $14.8 \%$ respectively). In the majority of the cases the villitis was mild.

CONCLUSION. In the population studied, the frequency of VUE is high (around 30\%). The lesion occurs in a similar frequency in placentas from NCP, PIH, SCH, SGA and AGA infants, but basal villitis not associated to a parenchymatous component affects mainly the placentas of NCP and AGA infants. It is possible that this kind of villitis could be an expression of an antigenic stimulation diverse from villitis with a parenchymatous component. [Rev Assoc Med Bras 2003; 49(I): 67-7I]

KeYworDs: Placenta. Villitis. Hypertension arterial. Small for gestational age infants.

\section{REFERÊNCIAS}

I. Fox H. Infections and inflammatory lesions of the placenta. In Fox H, editor. Pathology of the placenta. 2nd ed. London: W. B. Saunders; 1997. p.294-43.

2. RusselP. Infections of the placental villi (villitis). In: FoxH, editor. Haines and Taylor obstetrical and gynaecological pathology 3nd ed. Edinburgh: Churchill Livingstone; 1987. p. I0I4-29. 
3. Altshuler G, Russell P. The human placental villitides: a review of chronic intrauterine infection. Curr Top Pathol I 975; 60:63- I I 2 .

4. Labarrere $C$, Althabe O, Telenta M. Chronic villitis of unknown aetiology in placentae of idiopathic small for gestational age infants. Placenta I 982; 3:309-18,.

5. Labarrere C, Althabe $O$, Calenti E, Musculo D. Deficiency of blocking factors in intrauterine growth retardation and its relationship with chronic villitis. Am J Reprod Immunol Microbiol 1986; 10:14-9.

6. Laga EM, Driscoll SG, Munro HN. Comparison of placentas from two socioeconomic groups. I morphometry. Pediatrics 1972; 50:24-3 I.

7. KnoxWF, Fox H. Villitis of unknown aetiology: its incidence and significance in placentae from a british population. Placenta 1984; 5:395-402.

8. Redline RW, Abramowsky CR. Clinical and pathologic aspects of recurrent placental villitis. Hum Pathol I 985; |6:727-3|

9. Altemani A, Andrade LALA, Brenelli MA, Bacha AM. Vilosite placentária: correlação anátomoclínica.J Pediatria 1982; 52:315-8.

10. Russell P. Inflammatory lesions of the human placenta. The histopathology of villitis of unknown aetiology. Placenta 1980; I :227-44.

II. Nordenvall M, Sandstedt B. Placental villitis and intrauterine growth retardation in a Swedish population. Acta Pathol Microbiol Immunol Scand 1990; 98: 19-24.
12. Salafia CM, Pezzullo JC, Lopes-Zeno JA, Simmens S, MiniorVK, Vintzileos AM. Placental pathologic features of preterm preeclampsia. Am J Obstet Gynecol 1995; 173: 1097-105

13. Rüschoff J, Boger A, Zwiens G. Chronic placentitis: a clinicopathological study. Arch Gynecol 1 985; 237: 19-25.

14. Russell P. Inflammatory lesions of the human placenta. Villitis of unknown etiology in perspective. Am J Diagn Gynecol Obstet I 979; I:339-46.

15. Lubchenco LO, Searls DT, BrazieJV. Neonatal mortality rate: relatioshipi to birth weight and gestational age. J Pediatr 1972; 81:8I4-2.

16. Capurro H, Konichezki S, Fonseca D, Caldeyro-Barcia R. A simplified method for diagnosis of gestational age in the newborn infant.J Pediatr 1978; 93: 1 20-2.

17. Garcia AGP. Placental morphology of lowbirth-weight infants born at term: gross and microscopic study of 50 cases. Contrib Gynecol Obstet 1982; 9: 100- I 2

18. Nakamura Y, Sakuma S, Ohta Y, Kawano K, Hashimoto T. Detection of the human cytomegalovirus gene in placental chronic villitis by polymerase chain reaction. Hum Pathol 1994; 25:815-8.

19. Altemani AM, Fassoni A, Marba S. Cord IgM levels in placentas with villitis of unknown etiology. J Perinat Med 1989; 17:465-8.

20. Redline RW, Patterson P. Patterns of placental injury. Correlations with gestational age, pla- cental weight, and clinical diagnoses. Arch Pathol Lab Med I 994; I I 8:698-70 I.

21. Naeye RL. Disorders of the placenta, fetus, and neonate: diagnosis and clinical significance. St Louis: Mosby, 1992.

22. Altshuler G. A conceptual approach to placental pathology and pregnancy outcome. Semin Diagn Pathol 1993; 10:204-21.

23. Bjoro K, Myhre E. The role of chronic nonspecific inflammatory lesions of the placenta in intra-uterine growth retardation. Acta Pathol Microbiol Immunol Scand 1984; 92:133-7.

24. Mortimer G, MacDonald DJ, Smeeth A. A pilot study of the frequency and significance of placental villitis. Br J Obstet Gynaecol 1985; 92:629-33.

25. Redline RW, Patterson P. Villitis of unknown etiology is associated with major infiltration of fetal tissue by maternal inflammatory cells. Am J Pathol I993; 143:473-9.

26. Labarrere CA, Faulk WP. Maternal cells in chorionic villi from placentae of normal and abnormal pregnancies. Am J Reprod Immunol 1995; 33:54-9.

Artigo recebido: 05/02/2002 Aceito para publicação: 20/08/2002 